\title{
Avaliação da resistência à corrosão do aço AISI 420 depositado por processos variados de aspersão térmica
}

\author{
Corrosion resistance evaluation of AISI 420 steel deposited by various \\ thermal spray process
}

\section{Luiz Carlos Casteletti}

Escola de Engenharia de São Carlos EESC-USP, São Carlos - SP

E-mail: castelet@sc.usp.br

\section{Frederico Augusto Pires}

\section{Fernandes}

Escola de Engenharia de São Carlos EESC-USP, São Carlos - SP

E-mail:codoico@gmail.com

\section{Gustavo Satoru Takeya}

Escola de Engenharia de São Carlos EESC-USP, São Carlos - SP

E-mail: gutakeya@hotmail.com

\section{Carlos Alberto Picon \\ Faculdade de Engenharia de llha Solteira \\ FEIS-UNESP, Ilha Solteira - SP E-mail: capicone@dfq.feis.unesp.br}

\section{Germano Tremiliosi-Filho \\ Instituto de Química de São Carlos IQSC-USP, São Carlos - SP E-mail:germano@iqsc.usp.br}

\section{Resumo}

Entre as técnicas utilizadas no melhoramento de desempenho de materiais, a deposição na superfície de componentes pode ser eficaz para recuperação de elementos desgastados. Os processos de aspersão térmica, devido a sua versatilidade, quanto à aplicação, tipos de camadas e espessuras, constituem-se numa forma bastante adequada na obtenção de camadas de elevada dureza para proteção ou reparo do componente base, sendo possível revestir substratos metálicos com polímeros, metais ou cerâmicas. Entre esses processos, destacam-se: HVOF (High Velocity Oxygen Fuel), Arc-spray (arco elétrico) e Flame-spray (chama-pó). A seleção de um aço inoxidável, para aplicação, envolve algumas considerações como resistência à corrosão da liga, propriedades mecânicas, fabricabilidade e custo. Nesse trabalho foram usadas amostras do aço AISI 1045, revestidas, com aço inoxidável AISI 420, por meio das técnicas de Arc-Spray, HVOF e FlameSpray, para o estudo comparativo de suas resistências à corrosão em água do mar, visando à produção de peças de menor custo, em comparação com peças maciças desse aço. Os melhores desempenhos em termos de dureza, porosidade e resistência à corrosão ocorreram na seguinte seqüência crescente: Flame-Spray, Arc-Spray, e HVOF.

Palavras-chave: Aspersão térmica, corrosão, AISI 420.

\begin{abstract}
Among the techniques used to improve materials performance, deposition on the surface of components is a proper way of recovering worn elements. Thermal spraying processes were developed during the last few years and they are a very suitable method to obtain layers with high hardness for protecting or repairing the base component. Employing these processes, it is possible to overlay metallic substrates with polymers, metals and ceramics. Among these processes are: HVOF, Arc-Spray and Flame-Spray. The selection of a particular type of stainless steel for an application involves some considerations, as the corrosion resistance of the alloy, mechanical properties, manufacture feasibility and cost. In this work, used were samples of AISI 1045 steel, coated with stainless steel AISI 420, using the techniques of Arc-Spray, HVOF and Flame-Spray for the comparative study of their corrosion resistance in sea water, aimed at producing low-cost alternative pieces, compared with massive pieces of steel. The best performances in terms of hardness, porosity levels and corrosion resistance of the layers occurred in the following sequence growing: Flame-Spray, Arc-Spray, and HVOF.
\end{abstract}

Keywords: Thermal spray, corrosion, AISI 420. 


\section{Introdução}

O desenvolvimento de novos materiais e processos é um dos principais desafios tecnológicos industriais, uma vez que ocorre uma demanda incessante por componentes que atendam às necessidades crescentes quanto à resistência ao desgaste e/ou a corrosão, com o menor custo possível.

Entre as várias técnicas utilizadas no desenvolvimento de novos materiais, encontra-se a deposição de materiais, com características superiores às do metal base, nas superfícies dos componentes, melhorando, assim, seu desempenho quanto ao desgaste e/ou à corrosão, além de ser uma maneira eficiente para a recuperação de elementos danificados, acarretando diminuição de custos (Shipway; Mccartney; Sudaprasert, 2005; Souza; Neville, 2005; Gil; Staia, 1999). Os processos de aspersão térmica, devido a sua versatilidade quanto à aplicação e os tipos de camadas produzidas e suas espessuras, são uma das formas de se obter camadas de elevadas durezas para a proteção ou reparo do componente base. Nesses processos, é possível revestir substratos metálicos com polímeros, metal ou cerâmica. Entre esses processos, destacam-se: HVOF (High Velocity Oxygen Fuel) (Shipway; Mccartney; Sudaprasert, 2005; Scrivani et al., 2001; Sidhu; Agrawal; Prakash, 2005), Arcspray (arco elétrico) (Newbery; Grant; Neiser, 2005) e Flame-spray (chama-pó) (Gedzevicius; Valiulis, 2006; Lin; Han, 1998; Guo et al., 1995).

Vários trabalhos têm estudado o comportamento sob corrosão em água do mar de materiais depositados por aspersão térmica, os mais comuns são os cermets e ligas contra corrosão (Collazo; Nóvoa; Pérez, 1999).

Esse trabalho tem como objetivo analisar e comparar o comportamento, sob corrosão em água do mar natural, de revestimentos de aço inoxidável AISI 420 aplicado por três diferentes processos de aspersão térmica: Arc-spray, Flame-spray e HVOF, sobre substrato de aço AISI 1045.

\section{Materiais e métodos}

Foram usados corpos-de-prova com camadas de revestimentos de aço inoxidável AISI 420 sobre o aço AISI 1045, sendo que as camadas foram depositadas pelos seguintes processos: Arc-Spray, Flame-Spray e HVOF.

Para os revestimentos, foi utilizado pó do aço inoxidável AISI 420, cuja composição química nominal está apresentada na Tabela 1.

As medidas de dureza foram realizadas em um equipamento BUEHLER com carga de $100 \mathrm{gf}$ aplicada por $10 \mathrm{~s}$.

As camadas obtidas foram fotomicrografadas após preparação micrográfica convencional, com lixamento e polimento. Não foi usado ataque para a observação.

As medidas de porosidade foram efetuadas de modo automático, utilizando-se um software, de acordo com a norma ASTM E 2109.

A célula eletroquímica utilizada, para a obtenção das curvas de polarização potenciostáticas, consta de um eletrodo de referência de calomelano saturado (ECS) e um eletrodo auxiliar de platina. O eletrólito empregado foi água do mar natural (PH 8,0) obtida em alto mar da praia da Boa Viagem em Recife-PE. Foi empregado, também, um potenciostato modelo Autolab-VGSTAT

Tabela 1 - Composição química do material utilizado.

\begin{tabular}{c|c|c|c|c|c}
\hline \multirow{2}{*}{ Material } & \multicolumn{5}{|c}{$\%$ Peso dos elementos } \\
\cline { 2 - 6 } & C & Mn & Si & Cr & Fe \\
\hline AISI 420 & 0,15 & 1 & 1 & 13 & Bal. \\
\hline
\end{tabular}

Tabela 2 - Resultados de dureza Vickers e porosidade das amostras.

\begin{tabular}{c|c|c|c}
\hline \multirow{2}{*}{ Parâmetro } & \multicolumn{3}{|c}{ Amostra } \\
\cline { 2 - 4 } & Arc-Spray & Flame-Spray & HVOF \\
\hline Dureza, Hv & $544(80)$ & $414(75)$ & $615(55)$ \\
\hline Porosidade,\% & $13(5)$ & $17(3)$ & $6(2)$ \\
\hline
\end{tabular}

- 302, para monitoramento do potencial e corrente. As curvas de polarização anódicas dos aços foram obtidas com uma velocidade de varredura de $1 \mathrm{mV} / \mathrm{s}$. As correntes de corrosão foram calculadas no intercruzamento das curvas anódica e catódica linearizadas, na região do potencial de corrosão.

\section{Resultados e discussão}

Na Figura 1, são apresentadas micrografias ópticas da seção transversal de cada processo de aspersão. Por meio das micrografias, percebem-se diferenças significativas entre os métodos empregados, tais como o tamanho da partícula aspergida.

Na Tabela 2, apresentam-se os resultados de dureza média e porosidade das camadas obtidas nos três processos de aspersão.

Por meio da Tabela 2, pode-se observar que, pelo processo HVOF, conseguiu-se um revestimento com a maior dureza e menor porosidade, indicando que a porosidade influencia, negativamente, na dureza final da camada.

Na Figura 2, constam as curvas de polarização potenciodinâmicas referentes aos ensaios das três camadas e do substrato. A curva obtida para o material depositado pelo processo HVOF 


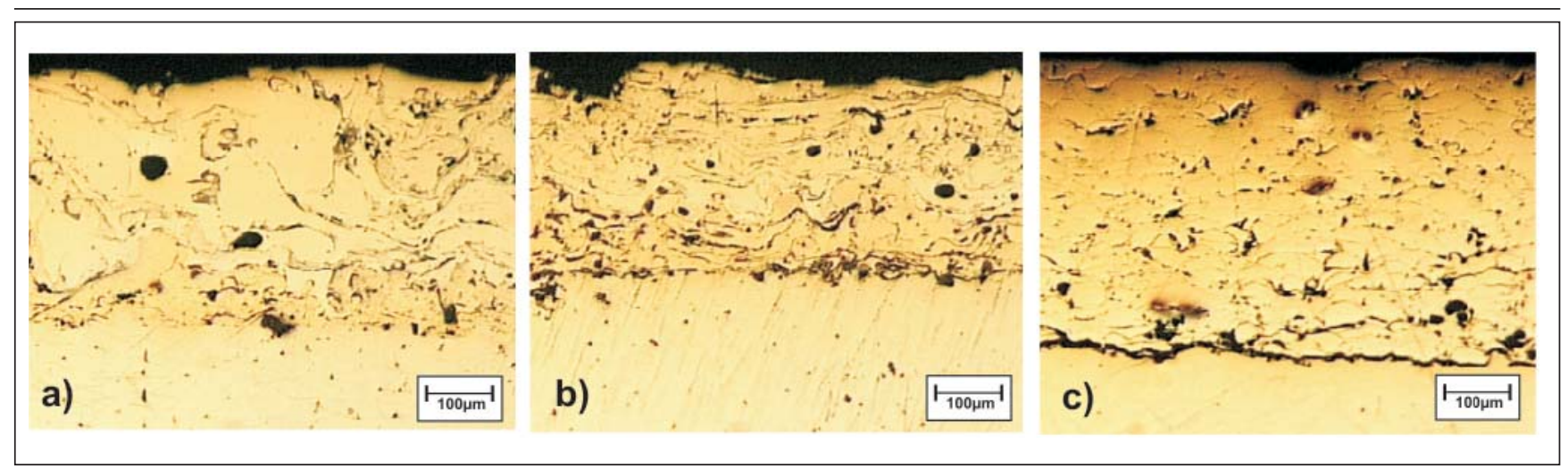

Figura 1 - Camada do aço inoxidável AISI 420 depositada pelo processo: a) Arc-Spray; b) Flame-Spray; c) HVOF.

apresenta o potencial de corrosão mais anódico (-492mV), quando comparado com os revestimentos obtidos pelos processos Arc-Spray (-528mV) e FlameSpray $(-547 \mathrm{mV})$. Observa-se, ainda, que as camadas depositadas pelo diferentes processos, apresentam um potencial de corrosão situado entre 150 e $200 \mathrm{mV}$, o qual é muito maior que o potencial de corrosão do substrato $(-696 \mathrm{mV})$, indicando uma boa proteção para o substrato.

Na Tabela 3, são apresentados os parâmetros coletados a partir das curvas de corrosão do substrato e para cada uma das amostras recobertas pelos métodos de aspersão empregados. Com base nos valores conjuntos do potencial e da corrente de corrosão, pode-se inferir que a camada obtida pelo processo HVOF apresenta o melhor desempenho frente à corrosão. As demais camadas, apesar de apresentaram um potencial de corrosão maior que o do substrato, exibem maiores correntes de corrosão.

Na Figura 3, são apresentadas as imagens obtidas por microscopia óptica

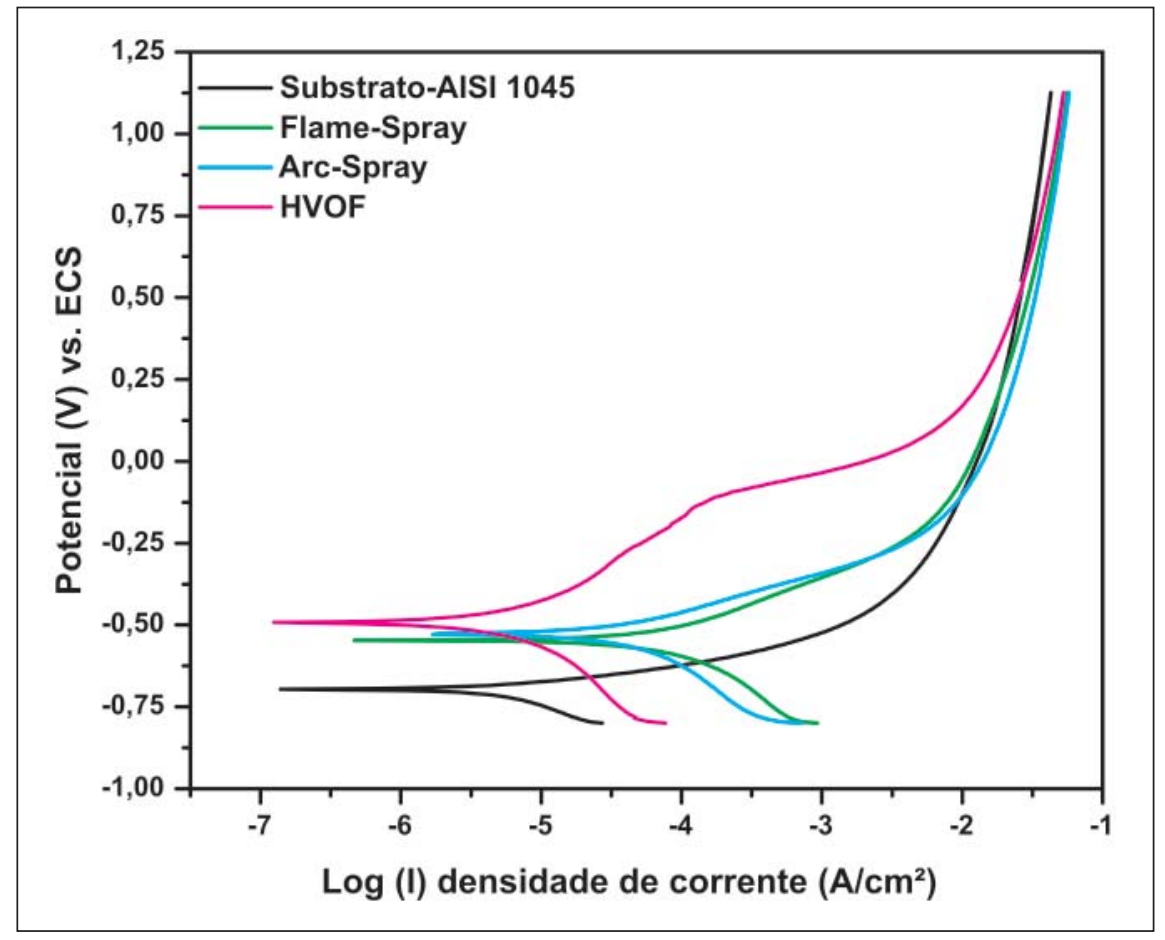

Figura 2 - Curvas de polarização potenciodinâmicas das amostras.

das superfícies resultantes da corrosão em água do mar para as camadas produzidas por cada um dos métodos de aspersão empregados.
Verifica-se que o processo corrosivo foi mais intenso no caso da camada produzida por Arc-spray, apesar de seu potencial de corrosão ser semelhante ao

Tabela 3 - Potenciais e correntes de corrosão das amostras estudadas.

\begin{tabular}{c|c|c|c|c}
\hline \multirow{2}{*}{ Parâmetro } & \multicolumn{4}{|c}{ Amostra } \\
\cline { 2 - 5 } & Substrato & Arc-Spray & Flame-Spray & HVOF \\
\hline Potencial de corrosão, $\mathbf{m V}$ & -696 & -528 & -547 & -492 \\
\hline Corrente de corrosão $\mathbf{\times 1 \mathbf { 1 0 } ^ { - 7 } , \mathbf { A } / \mathbf { c m } ^ { 2 }}$ & 1,4 & 16,9 & 4,7 & 1,2 \\
\hline
\end{tabular}



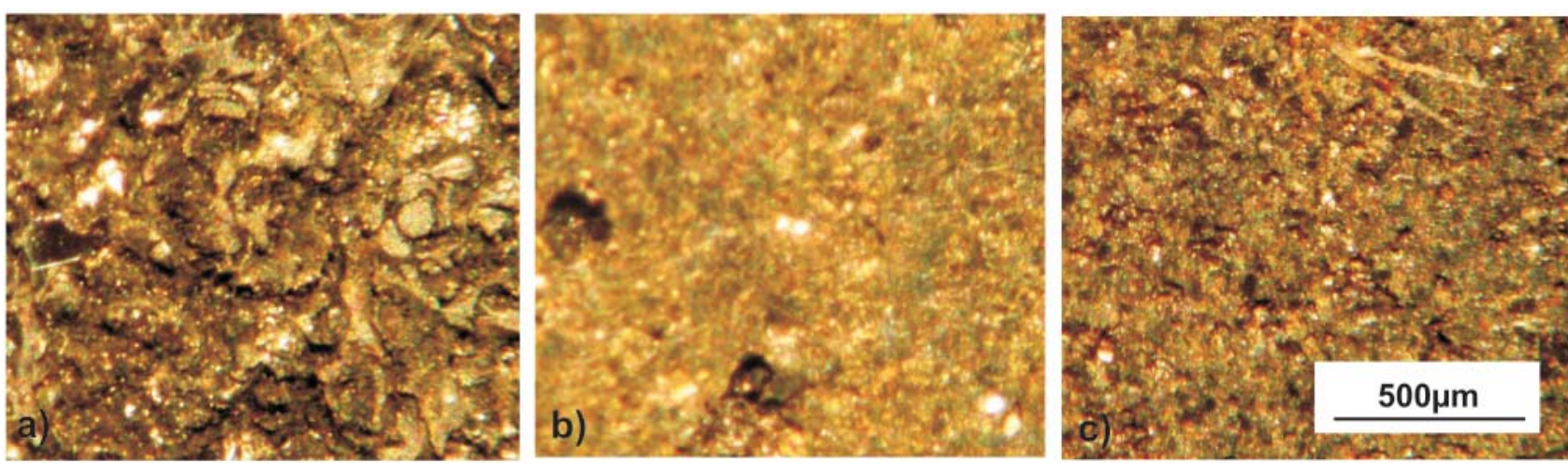

Figura 3 - Aspecto superficial dos recobrimentos após teste de corrosão: a) Arc-Spray; b) Flame-Spray; c) HVOF.

da camada obtida por Flame-spray. Entretanto, sua corrente de corrosão é cerca de quatro vezes maior, acentuando, significativamente, o processo corrosivo.

\section{Conclusões}

Foi observado que, entre os métodos de aspersão empregados, o processo por HVOF resultou em um revestimento com a maior dureza e menor nível de porosidade, indicando que a dureza está associada ao índice de porosidade e à maior resistência à corrosão.

Os revestimentos produzidos por Arc-spray e Flamespray apresentaram desempenhos intermediários entre os do revestimento HVOF e o do substrato.

Portanto a deposição por aspersão térmica de pó do aço inoxidável AISI 420 constitui-se em uma maneira bastante promissora de produção de peças resistentes à corrosão a custos inferiores aos das peças maciças do material.

\section{Agradecimentos}

Os autores agradecem a CAPES pela bolsa de estudo concedida a Frederico Augusto Pires Fernandes e ao CNPq (proc. 150799/2008-0).

\section{Referências bibliográficas}

COLLAZO, A., NÓVOA, X.R., PÉREZ, C. Corrosion behaviour of cermet coatings in artificial seawater. Electrochimica Acta, v.44, p. 4289-4296, 1999.
GEDZEVICIUS, I., VALIULIS, A. V. Analysis of wire arc spraying process variables on coatings properties. Journal of Materials Processing Technology, v. 175, n. 1-3, p. 206-211, 2006.

GIL, L., STAIA, M. H. Microstructure and properties of HVOF thermal sprayed NiWCrBSi coatings. Surface and Coatings Technology, v. 120-121, p. 423-429, 1999.

GUO, D. Z. et al. Effects of post-coating processing on structure and erosive wear characteristics of flame and plasma spray coatings. Surface and Coatings Technology, v. 73, n. 1-2, p. 73-78, 1995.

LIN, L., HAN, K. Optimization of surface properties by flame spray coating and boriding. Surface and Coatings Technology, v. 106, n. 2-3, p. 100-105, 1998.

NEWBERY, A. P., GRANT, P. S., NEISER, R. A. The velocity and temperature of steel droplets during electric arc spraying. Surface and Coatings Technology, v. 195, n. 1-3, p. 91-101, 2005.

SCRIVANI, A. et al. A contribution to the surface analysis and characterisation of HVOF coatings for petrochemical application. Wear, v. 250, n. 1-12, p. 107-113, 2001.

SHIPWAY, P. H., MCCARTNEY, D. G., SUDAPRASERT, T. Sliding wear behaviour of conventional and nanostructured HVOF sprayed WC-Co coatings. Wear, v. 259, n. 7-12, p. 820-827, 2005.

SIDHU, T. S., AGRAWAL, R. D., PRAKASH, S. Hot corrosion of some superalloys and role of high-velocity oxy-fuel spray coatings-a review. Surface and Coatings Technology, v. 198, n. 1-3, p. 441-446, 2005.

SOUZA, V. A. D., NEVILLE, A. Corrosion and synergy in a WCCoCr HVOF thermal spray coating understanding their role in erosion-corrosion degradation. Wear, v. 259, n. 1-6, p. 171-180, 2005.

Artigo recebido em 08/06/2009 e aprovado em $18 / 01 / 2010$.

\section{REM - Revista Escola de Minas www.rem.com.br}

